\title{
Contrast enhancement using recursive mean-separate histogram equalization for scalable brightness preservation
}

\begin{abstract}
Histogram equalization (HE) is widely used for contrast enhancement. However, it tends to change the brightness of an image and hence, not suitable for consumer electronic products, where preserving the original brightness is essential to avoid annoying artifacts. Bi-histogram equalization (BBHE) has been proposed and analyzed mathematically that it can preserve the original brightness to a certain extend. However, there are still cases that are not handled well by BBHE, as they require higher degree of preservation. This paper proposes a generalization of BBHE referred to as recursive mean-separate histogram equalization (RMSHE) to provide not only better but also scalable brightness preservation. BBHE separates the input image's histogram into two based on its mean before equalizing them independently. While the separation is done only once in BBHE, this paper proposes to perform the separation recursively; separate each new histogram further based on their respective mean. It is analyzed mathematically that the output image's mean brightness will converge to the input image's mean brightness as the number of recursive mean separation increases. Besides, the recursive nature of RMSHE also allows scalable brightness preservation, which is very useful in consumer electronics. Simulation results show that the cases which are not handled well by $\mathrm{HE}, \mathrm{BBHE}$ and dualistic sub image histogram equalization (DSIHE), have been properly enhanced by RMSHE.
\end{abstract}

Keyword: Bi-histogram equalization; Dualistic sub-image; Histogram equalization; Recursive mean-separate; Scalable brightness preservation 